\title{
Modeling, Calibration, and Evaluation of a Tendon-Actuated Planar Parallel Continuum Robot
}

\author{
Kathrin Nuelle ${ }^{1+}$, Tim Sterneck ${ }^{1+}$, Sven Lilge ${ }^{2}$, Dezhu Xiong ${ }^{1}$, Jessica Burgner-Kahrs ${ }^{2}$, Senior Member, IEEE \\ and Tobias Ortmaier ${ }^{1}$
}

\begin{abstract}
In this work, a novel planar parallel continuum robot $(\mathrm{PCR})$ is introduced, consisting of three kinematic chains that are coupled at a triangular end-effector platform and include tendon-actuated continuum segments. The kinematics of the resulting structure are derived by adapting the descriptions for conventional planar parallel manipulators to include constant curvature bending of the utilized continuous segments. To account for friction and non-linear material effects, a data-driven model is used to relate tendon displacements and curvature of the utilized continuum segments. A calibration of the derived kinematic model is conducted to specifically represent the constructed prototype. This includes the calibration of geometric parameters for each kinematic chain and for the end-effector platform.

During evaluation, positioning repeatability of $1.0 \%$ in relation to one continuum segment length of the robot, and positioning accuracy of $1.4 \%$, are achieved. These results are comparable to commonly used kineto-static modeling approaches for PCR. The presented model achieves high path accuracies regarding the robot's end-effector pose in an open-loop control scenario.
\end{abstract}

Index Terms-Flexible Robots, Parallel Robots, Kinematics, Calibration and Identification

\section{INTRODUCTION}

$\mathbf{P}$ ARALLEL continuum robots (PCR) benefit from the inherent characteristics of continuum robots such as high compliance, flexibility and low weight. The parallel setup increases the stiffness compared to continuum robots, while maintaining an overall passive compliance [1]. In comparison to conventional parallel robots, PCR trade accuracy and load capacity for a higher compliance. Thus, PCR can be beneficial in several different applications. For instance, the inherent compliance can enable safe human robot interactions, operations close to humans as an alternative to compliant control schemes, and industrial inspections in enclosed environments. Furthermore, the compliance benefits in assembly, e. g., when handling fragile parts or in peg-in-hole assembly to avoid jamming. Lastly, PCR could potentially be utilized in minimal

Manuscript received: February 24, 2020; Revised: May 18, 2020; Accepted June 24, 2020.

This paper was recommended for publication by Editor Dezhen Song upon evaluation of the Associate Editor and Reviewers' comments. This work was supported by the German Research Foundation (DFG) under grant no. OR 196/35-1 and BU 2935/5-1.

${ }^{1}$ Kathrin Nuelle, Tim Sterneck, Dezhu Xiong and Tobias Ortmaier are with the Leibniz University Hannover, Institute of Mechatronic Systems, An der Universität 1, 30823 Garbsen, Germany nuelledimes. uni-hannover.de

${ }^{2}$ Sven Lilge and Jessica Burgner-Kahrs are with the University of Toronto, Continuum Robotics Laboratory, 3359 Mississauga Road, Mississauga, ON L5L 1C6, Canada sven. lilge@utoronto. ca

${ }^{+}$Kathrin Nuelle and Tim Sterneck contributed equally.

Digital Object Identifier (DOI): see top of this page.

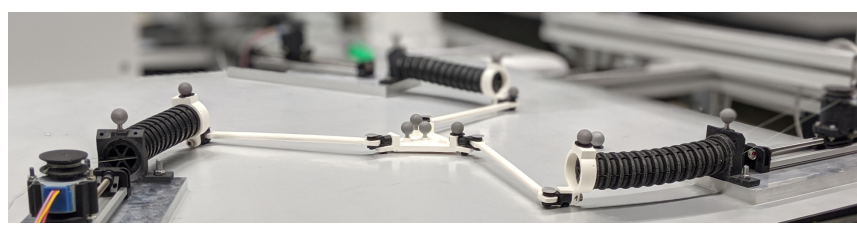

Fig. 1. Prototype of the proposed planar parallel continuum robot

invasive surgery, which is one of the major envisioned application for continuum robots in general. Instead of a one manipulator, multiple chains can enter the patient's body through small cuts or natural orifices before being physically coupled to form a parallel structure. The combination of multiple arms increases the system's stiffness and allows exerting higher manipulation forces to the environment in comparison to a single continuum robot. To investigate kinematic characteristics of PCR, this work introduces a continuum counterpart to the conventional 3-RRR parallel robot design with active continuum components in the robot's legs (see Fig. 1).

\section{A. Related works}

In the following, the most relevant PCR designs are discussed. This section is structured by the resulting actuationbased movements of the continuum robots within the PCRs.

1) Leg length-varying PCRs: Recent PCR research includes different continuum versions of a parallel Steward-Gough platform, utilizing length-changing elastic rods physically coupled at a common end-effector platform [2]-[5]. The resulting PCR offers six degrees of freedom (DoF). The kineto-static model of the elastic rod's deformation bases on Cosserat theory [6]. In [3], an experimental evaluation of pose accuracy and repeatability is presented, while a framework for modeling, analysis, and actuation-based force sensing is provided [7]. A constrained PCR is proposed in [8], coupling the legs by three intermediate platforms equally distributed along the rods. Three of its six flexible rods are actuated by varying their length. The robot is modeled using Euler-Bernoulli beam theory and the parameters of a set of non-linear equations are identified by using artificial neural networks. In [9], the employed beam theory model is extended using Cosserat rod theory. Further, a calibration technique for constrained PCR is proposed and experimentally validated. A planar 2-DoF PCR is introduced in [10], consisting of two elastic length-varying legs coupled at a common end-effector platform. A redundant, pseudo rigid link model for shape approximation is employed. A neuronal network is used to solve the inverse kinematics, as there exists no closed-form solution [11]. 
2) Bending and leg length-varying PCRs: Pneumatically actuated two-segment continuum segments are integrated in PCRs with two and three kinematic chains [12]. The actuators actively bend and vary its leg length while coupled to the end-effector platform by spherical joints. A constant curvature approach is employed for kinematic modeling and the design is optimized to increase the workspace size.

3) Passive continuum PCRs: A compliant Delta robot with spatial workspace is introduced in [13]. Each of the three robot chains consists of a rigid link with two mechanically coupled passive continuum segments at its ends. A kinematic model is derived using constant curvature assumptions to describe the continuum joints. The end-effector positioning accuracy is evaluated experimentally. In [14] and [15], a planar 2DoF mechanism utilizing passive continuous components is investigated. It consists of two compliant legs, coupled at a common end-effector using a revolute joint. For forward and inverse modeling, beam theory according to Euler-Bernoulli as well as Kirchhoff rod theory are applied to describe the flexible links. All solutions to the inverse kinematics problem are provided by interval Analysis in [16]. Several variations of the design are studied in [17], while one design is manufactured and experimentally verified [18]. The planar PCR proposed in [19] consist of a rigid end-effector coupled to two links, one flexible and one rigid. The developed kineto-static model describes the bending behavior of the PCR, discretizing the flexible link into 50 rigid links connected by elastic joints. A similar 3-DoF planar, flexible parallel robot is proposed in [20]. Again, modeling is based on a discretization approach and is validated experimentally in terms of positioning accuracy and repeatability.

Concluding, three different actuation implementations for PCR are proposed: (a) prismatic actuation of highly-elastic, length-changing legs, (b) a combination of bending and leg length-variation by pneumatic actuation, and (c) passive continuum segments with constant length, utilizing classical robotic actuation in other joints. Though, the commonly used tendon-actuation in continuum robotics have not been proposed in PCR designs, yet. In addition, modeling approaches for PCR mainly base on kineto-static based assumptions, describing the robot either in discretized lumped parameter form or as a deformable rod or beam. Simpler geometry based constant curvature approaches, which assume that bending of continuous backbone occurs in constant curvature arcs [21], are used in only a few publications for either passive continuum segments [13] or solely in modeling [12]. Active continuum components in PCR have not been evaluated using the constant curvature assumption.

\section{B. Contribution}

In this paper, we present a novel, tendon-actuated PCR design (see Fig. 2) as continuum counterpart to the conventional 3-RRR parallel robot. Due to the planar workspace, this structure is well suited for investigating kinematics and motion behavior of actively bending components within a parallel setup. So far, actively bending continuum segments with fixed lengths are only studied in mircometer-sized parallel structures, actuated by polymeric actuators [22]. Our work shows that it can also be used in larger scale PCRs, employing tendon-based actuation.

To model the kinematics, we develop a geometric model based on a constant curvature assumption, following the paradigm in [23]. In a robot-dependent step, a data driven model is employed to realistically relate tendon displacements of continuum segments to its resulting constant bending curvature. In the robot-independent step, the curvatures of each continuum segment are related to the pose of the end-effector platform using geometric assumptions. In comparison to most kineto-static models, this offers the advantage of applying methods from conventional parallel robots as their kinematic descriptions often rely of geometric assumptions. In particular, this allows Jacobian-based differential analysis. Furthermore, it makes our proposed structure easily comparable to conventional parallel robots.

The constant curvature approach usually excludes external forces, but the parallel setup induces coupling forces into the continuous components. Consequently, the proposed model can be seen as motion approximation. Its accuracy needs to be determined in order to utilized it in its potential application in an automatic control structures. To do so, we calibrate our kinematic model in a two-step procedure to fit it to the real robotic prototype. Lastly, we evaluate the repeatability of the prototype and the accuracy of the calibrated model in an unloaded open-loop scenario.

The main contribution of this paper consists of the evaluation of the proposed constant curvature based kinematic model using a real robotic prototype. Even though, this model is relatively simple in comparison to the vast majority of PCR kineto-statics based kinematic models, results show that the achieved accuracies are on par.

\section{ROBOT PROTOTYPE}

In this section, we first describe the robot design, followed by our modeling approach. With the kinematic model, we optimize the robot's workspace size and shape which mainly depends on the base locations and the joint limits.

\section{A. Robot design}

The kinematic structure of the proposed PCR design is mainly based on the commonly known planar 3-RRR parallel robot (see [24] e.g.). To derive our design, we substitute the proximal rigid link of each chain and the actuated revolute joint at its base by a tendon actuated continuum robot. An overview of the resulting structure and geometrical details are shown in Fig. 2. The PCR consists of three identical serial kinematic chains that are physically coupled to the triangular end-effector platform. The position of the revolute joints on the platform $\mathrm{P}_{i}$ is determined by the radius $r_{\mathrm{p}, i}$ and angle $\theta_{\mathrm{p}, i}$ and act as coupling points to the corresponding serial chain $i \in\{1,2,3\}$ (see Fig. $2 \mathrm{~d}$ ). The actuation of the three kinematic chains allows translation and rotation of the endeffector platform in a plane, resulting in three DoF. Thus, the end-effector pose is described by $\boldsymbol{x}_{\mathrm{E}}=\left[x_{\mathrm{E}}, y_{\mathrm{E}}, \phi_{\mathrm{E}}\right]^{\mathrm{T}}$. Each continuum segment of length $l_{\mathrm{C} i}$ is rigidly attached to the 


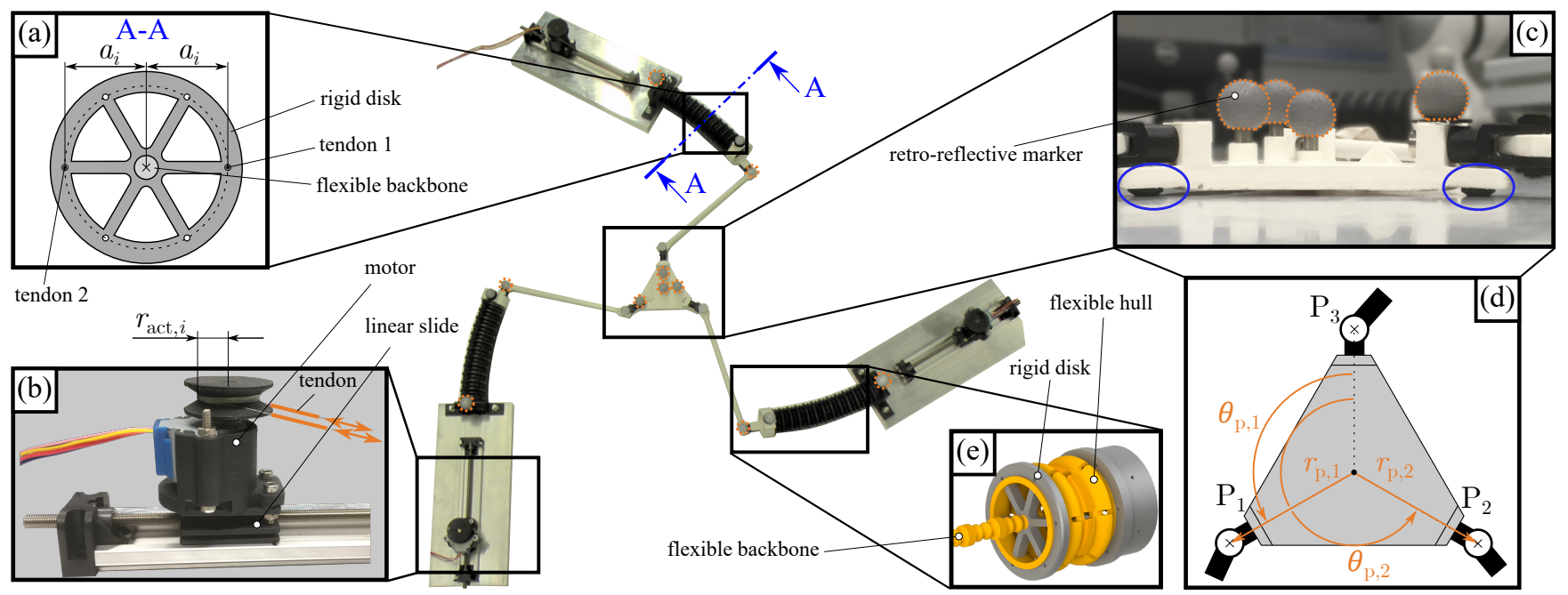

Fig. 2. Overview of prototype design and geometrical dimensions. Tendons of continuum robots are winded onto a common roll and actuated by a step motor as shown in (b). A linear guide enables adjustable pretension. Sliding guidance is seen in (c) in the form of surrounded contact points near to joints $\mathrm{P}_{i}$. Retro-reflective markers, used for localization measurements, are marked with dotted lines. Geometrical design of a continuum segment and end-effector platform are shown in (a) and (d), respectively, cuteaway rendering of the former consisting of flexible (orange) and rigid (gray) parts in (e).

base plate at the angle $\psi_{i}=\frac{2 \pi}{3}(i-1)$ and coupled to a rigid link of length $l_{\mathrm{st}, i}$ by a revolute joint $\mathrm{M}_{i}$. The bases $\mathrm{B}_{i}$ of each serial chain are equally distributed on a circle with a radius $r_{\text {Base }}$ that is optimized in Sec. II-C. Based on the taxonomy for parallel mechanisms by Merlet [24], we denote the kinematic structure of our proposed robot a $3-\mathrm{F} \underline{b} R R$, with $\mathrm{F} \underline{b}$ being a flexible continuum segment with constant length, using solely bending as degree of actuation.

Low weight and low friction joints (model GERMK-05, igus, Cologne, Germany) are chosen for all passive revolute joints. They offer a range between $-132^{\circ}$ and $132^{\circ}$. The rigid links, the end-effector platform, and the holding and coupling units were printed using polylactic acid (PLA). The continuum segments are fabricated in multi-material additive manufacturing, using a combination of rigid and highly elastic photopolymer material (Vero Black and Tango Black Plus, Stratasys, Eden Prairie, MN, USA). The backbone and hull (inspired from [25]) are flexible and shaped in bellows (see orange parts in $2 \mathrm{e}$, while gray parts are rigid). Six tendons can be routed through the rigid spacer disks (see Fig. 2a), allowing spacial bending and length-changing of the backbone for future prototypes. For the presented 3-FbRR robot, only two tendons are located at a distance $a_{i}$ on opposite sides of the backbone. Each tendon pair is winded onto a roll with radius $r_{\text {act }}$ (see Fig. 2b), that is actuated by one stepper motor (type 28BYJ-48), resulting in equal tendon displacements with opposing signs. The resulting motion capabilities of each continuum segments results in planar bending, limited to $180^{\circ}$ in order to avoid material fatigue. The stepper motors are mounted on a linear slide, enabling an adjustable preload to the tendons, backbone, and hull. The actuation rolls allow operating the robot with fixed length. To reduce friction during the planar motion on the smooth base plate lubricated bolt heads (see Fig. 2c) are attached at the bottom of all passive joints.

\section{B. Modeling}

The derived kinematics model is solely based on geometric assumptions. To approximate the motion behavior of the employed continuum segments, it is assumed that bending occurs with constant curvatures (Fig. 3a, see [23]). As stated above, the model is divided in a robot-dependent part, relating tendon displacements in a single continuum segment to its resulting bending curvature and in a robot-independent part, relating continuum segment curvatures to the end-effector platform pose.

a) Robot-dependent model: An arc with the length $l_{\mathrm{C} i}$ describes the geometry of the $i$-th continuum robot. The curvature $\kappa_{i}$ is the inverse of the arc's radius and yields

$$
\kappa_{i}=\frac{\Delta l_{i}}{a_{i} l_{\mathrm{C} i}}=\frac{q_{i} r_{\mathrm{act}, i}}{a_{i} l_{\mathrm{C} i}},
$$

where $\Delta l_{i}$ is the tendon displacement and $q_{i}$ is the position of the stepper motor driving the tendons. The mapping of curvature and tendon displacement will be represented by a data driven, non-linear model obtained during the calibration procedure, outlined in Sec. III-B.

b) Robot-independent model: The robot-independent model describes the robot kinematics with respect to the curvature $\kappa_{i}$, independently from the actuation principle, as described in [23]. The kinematics are derived based on the well-known analytical approach for conventional parallel manipulators according to [24]. Note that all coordinates and vectors are defined in the robot coordinate frame $(\mathrm{CF})_{0}$, unless it is specified as lower prefix in brackets. $(\mathrm{CF})_{0}$ is located in the center of the bases and its $x$-axis is aligned in parallel to $\boldsymbol{r}_{\mathrm{B}_{1}, \mathrm{~B}_{2}}$, the vector from Points $\mathrm{B}_{1}$ to $\mathrm{B}_{2}$. In the analytical approach, geometric constraints are defined that result from closing vector loops, building concatenated vectors between fixed base points $\mathrm{B}_{i}$ and coupling points $\mathrm{P}_{i}$ at the end-effector 


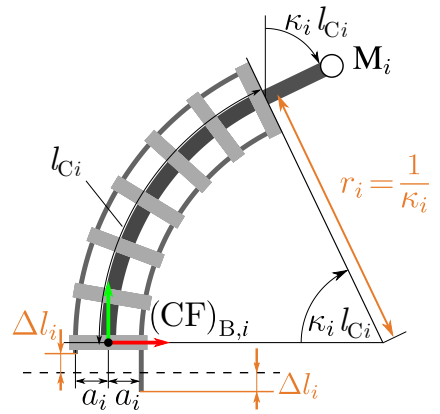

(a)

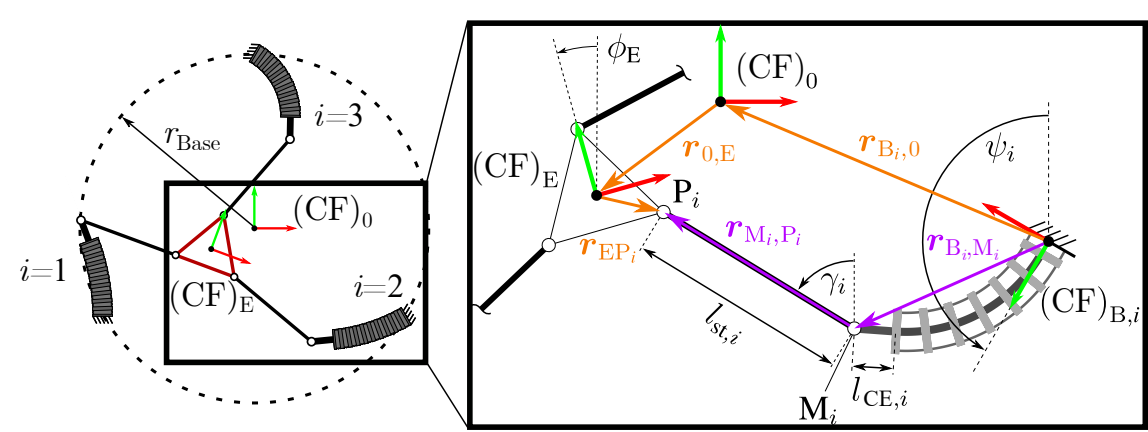

(b)

Fig. 3. The geometry parameters of an arc (a) are used to model the robot dependent kinematics of the continuum segments. The kinematics of entire parallel structure in the middle is described by vector chains defining the $i$-th kinematic chain (b).

platform for each serial kinematic chain $i$, shown in Fig. 3b. The geometric constraints of the parallel system result to

$$
\boldsymbol{r}_{\mathrm{B}_{i}, \mathrm{M}_{i}}+\boldsymbol{r}_{\mathrm{M}_{i}, \mathrm{P}_{i}}=\boldsymbol{r}_{\mathrm{B}_{i}, 0}+\boldsymbol{r}_{0, \mathrm{E}}+\boldsymbol{r}_{\mathrm{E}, \mathrm{P}_{i}} .
$$

The Vector $\boldsymbol{r}_{\mathrm{M}_{\mathrm{i}}, \mathrm{P}_{\mathrm{i}}}$ depends on the passive angle $\gamma_{i}$ at point $\mathbf{M}_{i}$. Rearranging (2) and applying the squared 2-norm yields

$$
\left\|\boldsymbol{r}_{\mathrm{M}_{i}, \mathrm{P}_{i}}\right\|_{2}^{2}=\left\|\boldsymbol{r}_{\mathrm{B}_{i}, 0}+\boldsymbol{r}_{0, \mathrm{E}}+\boldsymbol{r}_{\mathrm{E}, \mathrm{P}_{i}}-\boldsymbol{r}_{\mathrm{B}_{i}, \mathrm{M}_{i}}\right\|_{2}^{2} .
$$

The vector $\boldsymbol{r}_{\mathrm{B}_{i}, \mathrm{M}_{i}}$ results from the forward kinematics of the continuum robot, adding the translational displacement between the continuum robot's tip and joint $\mathrm{M}_{i}$ as following:

$$
\boldsymbol{r}_{\mathrm{B}_{\mathrm{i}}, \mathrm{M}_{\mathrm{i}}}=\boldsymbol{R}_{\psi_{i}}\left[\begin{array}{c}
\frac{1}{\kappa_{i}}\left(1-\cos \left(\kappa_{i} l_{\mathrm{C} i}\right)\right) \\
\frac{1}{\kappa_{i}} \sin \left(\kappa_{i} l_{\mathrm{C} i}\right)
\end{array}\right]+\boldsymbol{R}_{\psi_{i}} \boldsymbol{R}_{-\kappa_{i} l_{\mathrm{C} i}}\left[\begin{array}{c}
0 \\
l_{\mathrm{CE}, i}
\end{array}\right],
$$

where $\boldsymbol{R}_{*}$ is a rotation matrix for rotations around the $z$-axis with angle $*$. Rearranging leads to the three implicitly defined geometric constraints $h_{i}$ :

$$
\begin{aligned}
h_{i} & =\left\|\left[\begin{array}{l}
-\mathrm{B}_{x, i}+x_{\mathrm{E}} \\
-\mathrm{B}_{y, i}+y_{\mathrm{E}}
\end{array}\right]+\boldsymbol{R}_{\phi_{\mathrm{E}}}\left[\begin{array}{c}
(E) \mathrm{P}_{i, x} \\
{ }_{(E)} \mathrm{P}_{i, y}
\end{array}\right]-\boldsymbol{r}_{\mathrm{B}_{i}, \mathrm{M}_{i}}\right\|_{2}^{2}-l_{\mathrm{st}, i}^{2} \\
& \stackrel{!}{=} 0
\end{aligned}
$$

which are solved numerically to get the curvature

$$
\kappa_{i}=f_{\mathrm{IK}}\left(h_{i}\right)=\arg \min _{\kappa_{i}}\left\|h_{i}\right\|_{2}^{2}
$$

in case of inverse kinematics or end-effector pose

$$
\boldsymbol{x}_{\mathrm{E}}=\boldsymbol{f}_{\mathrm{DK}}(\boldsymbol{h})=\arg \min _{\boldsymbol{x}_{\mathrm{E}}}\|\boldsymbol{h}\|_{2}^{2}
$$

with $\boldsymbol{h}=\left(h_{1}, h_{2}, h_{3}\right)^{\mathrm{T}}$ in case of forward kinematics, due to the absence of an analytical closed form solution. Limitations of the joint angles and the bending curvatures were considered as additional constraints.

\section{Workspace enhancement}

The size of the robot's workspace mostly depends on the base radius $r_{\text {Base. }}$ The CAD parameters $\left(r_{\mathrm{p}, i}=59 \mathrm{~mm}\right.$, $\left.\theta_{\mathrm{p}, i}=\frac{2 \pi}{3} i, l_{\mathrm{C} i}=125 \mathrm{~mm}, l_{\mathrm{st}, i}=164 \mathrm{~mm}, l_{\mathrm{CE}, i}=36 \mathrm{~mm}\right)$ are chosen to create a tabletop-size robot for experimental model validation in Sec. III. Additionally, a major limitation in terms of workspace size and shape is the range of the employed joint angles. To optimize the workspace, we

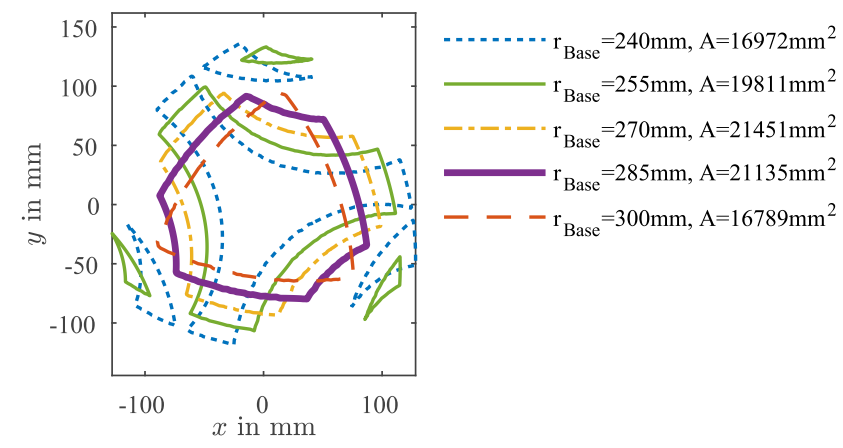

Fig. 4. Workspace contours for various values of the base radius $r_{\text {Base }}$. The $0^{\circ}$ translational workspace of the PCR was obtained by discretizing the joint space and intersecting the leg workspaces which result from applying alpha shapes to the discretized point clouds.

consider different base radii $r_{\text {Base }}$ to find a trade-off between its size and appropriate shape. The translational workspace is calculated by intersecting all leg workspaces of the PCR. A leg workspace is determined by discretizing the active and passive joints and calculating the resulting end-effector position with given orientation $\phi_{\mathrm{E}}$, creating point clouds for each leg. Only points within a circle around $(\mathrm{CF})_{0}$ with the radius $l_{\mathrm{st}, i}+l_{\mathrm{C} i}$ are considered to reduce the number of points. An enclosing area, the leg workspace, is created using alpha shapes [26] ( $\alpha=5)$. An intersection point cloud results from checking all points of each cloud, whether they lie within all leg workspaces. The resulting points are again combined into an alpha shape area that represents the translational workspace of the PCR. Workspace contours and size for different values of base radius are shown in Fig. 4. Note that the joint limitations lead to a rugged $0^{\circ}$ translational workspace for small values of $r_{\text {Base }}$. The joint restrictions also decrease the usable workspace into a very thin area for decreasing $r_{\text {Base }}$, while the outer limits expand further from the center. Considering the existing restrictions, we chose the final base radius to be $285 \mathrm{~mm}$ as a trade-off between workspace size and shape.

\section{EXPERIMENTAL VALIDATION}

The above described robot prototype was built to validate our proposed model and determine its accuracy. The employed trajectory planner and the open-loop control scheme 
is presented to generate all robot movements for evaluation. Followed by the detailed calibration procedure, the robot's repeatability, pose, and path accuracy is evaluated.

\section{A. Trajectory Planning and Open Loop Control}

A trajectory planner is implemented to handle discretetime motions in both, task and joint space, using the kinematic model described in the previous section. Trajectories are checked for violations of the workspace boundaries and singularities along the path. Singularities occur when the determinant of the analytically determined matrices $\boldsymbol{A}=\frac{\partial \boldsymbol{h}}{\partial \boldsymbol{x}_{\mathrm{E}}}$ or $\boldsymbol{B}=\frac{\partial \boldsymbol{h}}{\partial \boldsymbol{\kappa}}$ equals zero [24], using $\boldsymbol{h}$ of (5). To evaluate the prototype, linear, circular and rectangular paths where used. Multiple paths can be linked together with constant or trapezoidal velocity profiles.

An open-loop control is implemented in order to determine the accuracy of the proposed model. A microcontroller (Arduino $^{\mathrm{TM}}$ Uno) sets the desired positions for each implemented stepper motor. Motions of the motors are synchronized with fixed cycle time of $20 \mathrm{~ms}$, a safe cycle time where no data loss occurred.

\section{B. Calibration}

In kinematic or level two calibration [27], the geometric parameters of the robot are identified and the accuracy of the model is increased by parameter optimization. The proposed calibration procedure was inspired by the work in [28], where different measurements for external kinematic calibration of a 3-DOF planar parallel robot were investigated. Errors from deformations are relatively small in classical parallel robotics due to the rigid links. In contrast, the chains of PCR compose of highly flexible components that require special attention. Thus, the kinematic calibration process is decomposed into two steps. We assume that the continuum segments undergo constant curvature bending. However, a linear function, as presented by (1), has limitations describing the real relationship between the bending curvature $\kappa_{i}$ and the tendon displacement $\Delta l_{i}$, due to friction effects, hysteresis, non-linear material properties and varying material stress along the backbones. The latter results from the bellow shape and fabrication of the continuum segments, complicating the usage of existing methods as in [7]. Therefore, a regression function with the coefficients $k$ is used to describe this relationship, using the tracked joint positions $\mathrm{M}_{i}$ to calibrate the parameter vector $\boldsymbol{P}_{1}=\left[\boldsymbol{l}_{\mathrm{C}}, \boldsymbol{\psi}, \boldsymbol{k}\right]$ in the first step. The before mentioned parameters $a_{i}$ and $r_{\text {act, } i}$ are not calibrated, since they are no longer part of the robot specific kinematics. In the second step, the real position of the platform's joints in polar coordinates $\left(\boldsymbol{P}_{2}=\left[\boldsymbol{r}_{\mathrm{p}}, \boldsymbol{\theta}_{\mathrm{p}}\right]\right)$ are identified using the measured endeffector's pose $\boldsymbol{x}_{\mathbf{E}}$.

For the entire calibration procedure, the continuum's tip $l_{\mathrm{CE}, i}$ as well as the length of the straight link $l_{\mathrm{st}, i}$ are measued by calliper and the base positions $B$ by a tactile measuring arm (FARO Technologies Inc., Lake Mary, USA). To measure the exact pose of the robot prototype, we used a camerabased motion capturing system (model Oqus4, Qualysis AB,

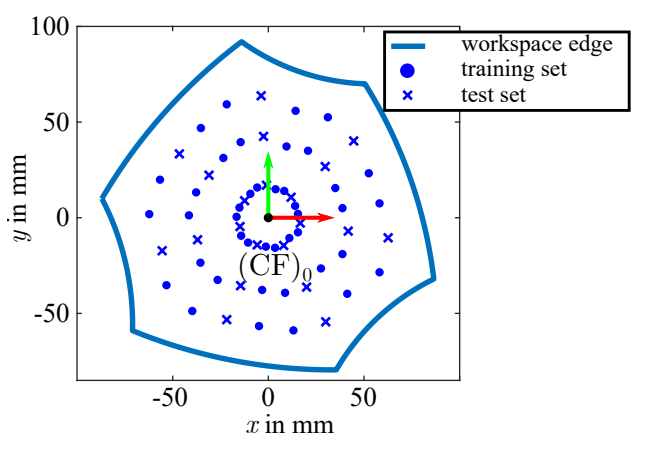

Fig. 5. Target positions used in the calibration procedure, resulting from equally-distributed points on differently scaled workspace edges. Circular marked poses marked the training set, while x-marked poses build up the test set.

Göteborg, Sweden) with a precision of $0.1 \mathrm{~mm}$. Six infrared sensitive cameras detect retro-reflective markers attached to the fixed base points and the moving end-effector platform. A stationary reference coordinate frame was defined using one marker at each continuum segment base in addition to one marker on the base plate itself. Four additional markers were placed at the end-effector platform and the joints $\mathrm{M}_{i}$ were also marked to decompose the calibration process. The locations of stationary markers were measured tactilely. A point-based registration of the base markers was performed to receive the transformation between the robots coordinate frame (calculated from the tactile measurements) and the optical marker frame.

We choose 63 equally distributed points along the scaled workspace boundaries for calibration, considering different scaling factors $(0.75,0.5$ and 0.2$)$, for three different constant orientations $\left(0^{\circ}, 10^{\circ}\right.$ and $\left.-10^{\circ}\right)$. No singularities occur in the translational workspace for the chosen orientations. Fig. 5 shows the selected end-effector positions for $\phi_{\mathrm{E}}=0^{\circ}$. The total of 189 poses are divided into a training set (126 poses) for calibration and a test set (63 poses) for validation. Our tested mapping functions, that are proposed for regression problems in [29], are shown in the second column of Tab. I. The cost

TABLE I

REMAINING ERROR ON THE TEST SET OF THE REGRESSION FUNCTIONS USED TO MAP THE RELATIONSHIP BETWEEN $\Delta l_{i}$ AND $\kappa_{i}$

\begin{tabular}{llc}
\hline Index & Regression function $\kappa_{i}$ & RMS in mm \\
\hline 1 & $k_{0, i} \Delta l_{i}$ & 1.57 \\
2 & $k_{0, i}+k_{1, i} \Delta l_{i}$ & 1.31 \\
3 & $k_{0, i}+k_{1, i} \Delta l_{i}+k_{2, i} \Delta l_{i}^{2}$ & 1.32 \\
4 & $k_{0, i}+k_{1, i} \Delta l_{i}+k_{2, i} \Delta l_{i}^{2}+k_{3, i} \Delta l_{i}^{3}$ & 1.31 \\
5 & $k_{0, i}+k_{1, i} \Delta l_{i}+k_{2, i} \Delta l^{1 / 2}$ & 1.24 \\
6 & $k_{0, i}+k_{1, i} \Delta l_{i}+k_{2, i} \Delta l^{1 / 2}+k_{3} \Delta l^{1 / 3}$ & 1.24 \\
7 & $k_{0, i}+k_{1, i} \Delta l_{i}+\ldots+k_{n, i} \Delta l^{1 / n}, n=4$ & 1.22 \\
8 & $k_{0, i}+k_{1, i} \Delta l_{i}+\ldots+k_{n, i} \Delta l^{1 / n}, n=5$ & 1.26 \\
9 & $k_{0, i}+k_{1, i} \Delta l_{i}+\ldots+k_{n, i} \Delta l^{1 / n}, n=6$ & 1.26 \\
10 & $k_{0, i} \ln \left(\Delta l_{i}\right)$ & 8.30 \\
11 & $k_{0, i}+k_{1, i} \ln \left(\Delta l_{i}\right)$ & 7.90 \\
12 & $k_{0, i}+k_{1, i} \Delta l+k_{2, i} \ln \left(\Delta l_{i}\right)$ & 1.24 \\
13 & $k_{0, i}+k_{1, i} \Delta l+k_{2, i} \Delta l^{2}+k_{3, i} \ln \left(\Delta l_{i}\right)$ & 1.24
\end{tabular}


function of the first calibration step is given as

$$
\min _{\boldsymbol{P}_{1}} \sum_{j=1}^{126} \sum_{i=1}^{3}\left(\hat{x}_{\mathrm{M}_{j, i}}-x_{\mathrm{M}_{j, i}}\right)^{2}+\left(\hat{y}_{\mathrm{M}_{j, i}}-y_{\mathrm{M}_{j, i}}\right)^{2},
$$

where $j$ denotes the index of training set. $\left(\hat{x}_{\mathrm{M}_{j, i}}, \hat{y}_{\mathrm{M}_{j, i}}\right)$ indicates the estimated position of joint $\mathrm{M}_{i}$ based on the regression model for given actuation $\Delta l_{j, i}$. The actual position $\left(x_{\mathrm{M}_{j, i}}, y_{\mathrm{M}_{j, i}}\right)$ is recorded. The model is solved using nonlinear least squares with a trust-region-reflective algorithm. Next to the tested regression functions, the remaining root mean squared (RMS) error of $\mathrm{M}_{i}$ on the test set is shown in Tab. I. After comparing the performance on the test set, function 7 performed best and is chosen to map $\Delta l_{i}$ and $\kappa_{i}$.

In the second calibration step, the already estimated parameters from the first part are set as constant. The cost function of this step is

$$
\min _{\boldsymbol{P}_{2}} \sum_{j=1}^{126}\left(\left(\hat{x}_{\mathrm{E}_{j}}-x_{\mathrm{E}_{j}}\right)^{2}+\left(\hat{y}_{\mathrm{E}_{j}}-y_{\mathrm{E}_{j}}\right)^{2}+\left(\hat{\phi}_{\mathrm{E}_{j}}-\phi_{\mathrm{E}_{j}}\right)^{2}\right)
$$

to determine $\boldsymbol{P}_{2} \cdot \hat{\boldsymbol{x}}_{\mathrm{E}_{j}}$ is estimated by the forward kinematic in (7) with the given $j$-th actuation values $\Delta l_{j}$.

\section{Evaluation}

All experimental investigations regarding performance indices were made using the optical measurement system described in Sec. III-B. Beside the studies conducted using the calibrated robot, all following evaluations regarding pose and path-following accuracy were determined before calibration as well. The complete results, comparing both in Table II, show the improvement due to the usage of the calibrated model.

a) Pose Repeatability: The repeatability of our prototype is evaluated in the first part of the experimental evaluation. The test procedure generally leans on ISO 9283, but specific different aspects are mentioned in the following. For this purpose, a new measurement of the 63 evenly distributed target poses with $\phi_{\mathrm{E}}=0^{\circ}$, taken from the calibration procedure (see Fig. 5), is recorded. Each of these poses was targeted 20 times, consecutively starting from workspace's origin $\boldsymbol{x}_{\mathrm{E}}=[0,0,0]^{\mathrm{T}}$ using trapezoidal velocity profiles. The real end-effector poses were measured simultaneously and the error was calculated as the difference between measured poses and their mean pose. Positioning and orientation repeatability is determined as $2 \sigma$ neighborhood of the test series (see ISO 9283) with standard deviation $\sigma$.

The positioning and orientation repeatability are $1.2 \mathrm{~mm}$ and $0.5^{\circ}$, respectively. The RMS error on the 63 poses is presented in Tab. II, column pose accuracy. The positioning repeatability corresponds to $1.0 \%$ of the nominal length of a continuum segment $l_{\mathrm{C} i}$. We chose $l_{\mathrm{C} i}$ as reference length, since all experimental results on PCR are presented in reference to the length of implemented continuous elements. The positioning repeatability at each measured pose is shown within translational workspace in the accompanying video attachment. b) Pose Accuracy: For determining the pose accuracy, the end-effector poses of the repeatability test were measured again and real poses compared to those, generated from calibrated robot model. Pose error consists of positioning error $\Delta r_{\mathrm{E}}$ and orientation error $\Delta \phi_{\mathrm{E}}$ as difference between target pose from model and measured pose (see ISO 9283). Characteristic values (mean value $\bar{x}$, maximum value $x_{\max }$ and standard deviation $x_{\sigma}$ ) for positioning and orientation accuracy over all measured end-effector poses are shown in Table II, column pose accuracy.

c) Open-Loop Path-Following Accuracy: Furthermore, the path-following accuracy is determined, using a circular trajectory in the workspace center with $50 \mathrm{~mm}$ radius, constant $0^{\circ}$ end-effector orientation and a constant path velocity of 50 $\mathrm{mm} / \mathrm{s}$. The latter is limited by the maximum drive speed, the former by workspace boundaries. The second tested trajectory was a square with width of $50 \mathrm{~mm}$ and constant orientation of $0^{\circ}$. In contrast to the circle, the linear parts were combined with trapezoidal velocity profiles with a maximum of $50 \mathrm{~mm} / \mathrm{s}$. Path error results from the difference between poses from trajectory planning at grid points in time and simultaneously measured end-effector poses to consider time dependency. Measured and planned paths are compared in Fig. 6. Extracted path errors regarding positioning $\left(\Delta r_{E}\right)$ and orientation of the end-effector $\left(\Delta \phi_{\mathrm{E}}\right)$ are shown in Tab. II, the columns for path accuracy. The path-following positioning accuracy is slightly higher and the orientation accuracy is slightly lower compared to pure static positioning. No specification for path accuracy of PCR structures are given in literature to the best of our knowledge.

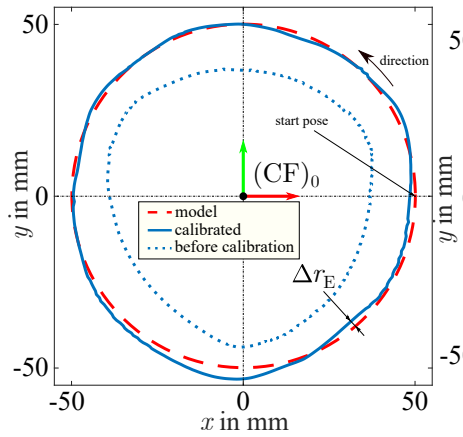

(a)

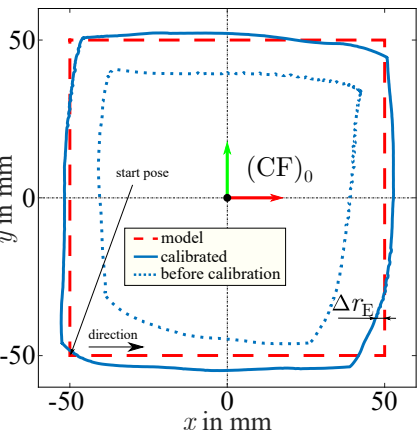

(b)
Fig. 6. Planned (red, dashed lines) and measured (blue, solid lines) endeffector path of the test trajectories are presented. The circular trajectory (a) was driven with constant velocity of $50 \mathrm{~mm} / \mathrm{s}$, the square trajectory (b) was build up from four linear rest-to-rest trajectories with trapezoidal velocity profile. Blue dot dash lines show measured paths before the kinematic calibration.

d) Effect of Gear Backlash on Positioning Accuracy:

Looking at the employed test trajectories and the achieved accuracies, it is noticeable that accuracy is worse on a square trajectory. One part of this inaccuracy can be traced back to a combination of stepper's gear backlash and static friction on the base plane. To model and account for these effects, the minimal detectable step number $\boldsymbol{n}_{\mathrm{S}}=\left[n_{\mathrm{S}, 1}, n_{\mathrm{S}, 2}, n_{\mathrm{S}, 3}\right]^{\mathrm{T}}$ of the motors after a change of direction was determined. To do so, the motors moved a specific number of steps forth and the same number back, while recording the optical 
TABLE II

EVALUATION RESULTS OF THE POSE AND PATH-FOLLOWING ACCURACY

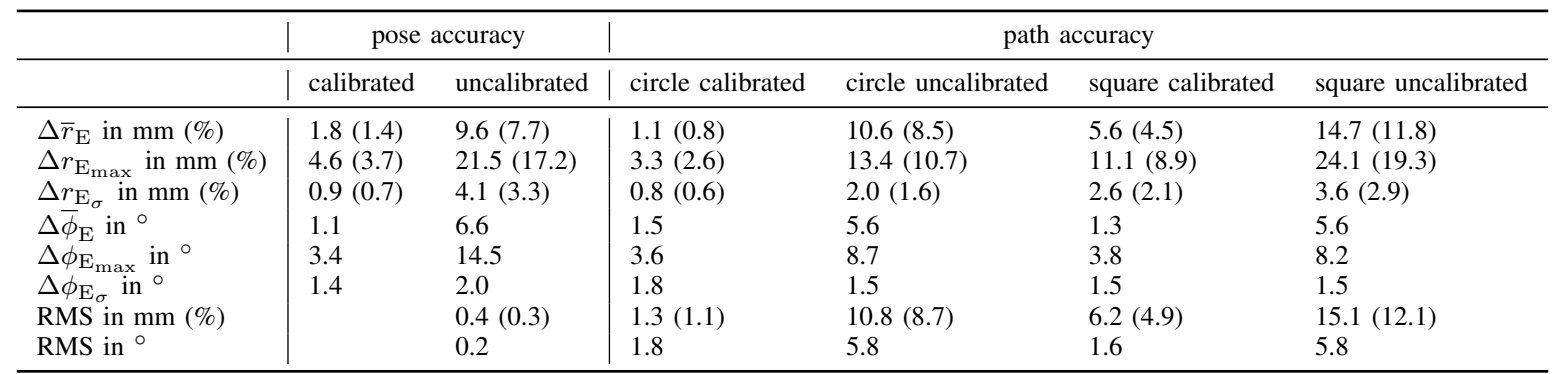

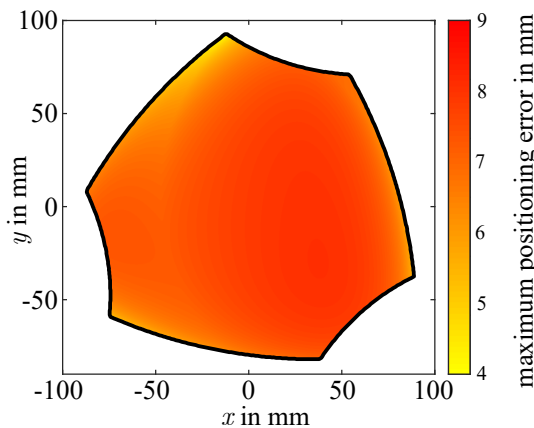

Fig. 7. Maximum positioning error in $0^{\circ}$-translational workspace resulting from gear backlash of step drives. The estimation is made using the Jacobian.

markers at the tip of the continuum robots. Incrementally, we increased the number of steps, starting by one, and performed a fast Fourier transformation on each measured position signal. When the amplitude at the movement's frequency (about $0.5 \mathrm{~Hz}$ ) exceeded the precision of the optical tracking system, $n_{\mathrm{S}, i}$ was recorded. The maximum resulting end-effector error is pose-depended and estimated by

$$
\Delta \boldsymbol{x}_{\mathrm{E}}=|\boldsymbol{J}| \Delta \boldsymbol{q}=|\boldsymbol{J}| \frac{2 \pi r_{\mathrm{act}}}{n_{\mathrm{spr}}} \boldsymbol{n}_{\mathrm{S}}
$$

with $\boldsymbol{J}=-\boldsymbol{A}^{-1} \boldsymbol{B}$ [24] being the robot's Jacobian and $n_{\mathrm{spr}}$ being the number of steps per rotation. The overall maximal positioning error, resulting from gear backlash and static friction, is shown in Fig. 7. Relatively high errors occur in the lower part of the workspace, close to its boundaries. That roughly corresponds to lower right corner $(50,-50)$ of the square trajectory, where the positioning error is the highest.

\section{DISCUSSION}

In this section, we discuss the achieved results in terms of positioning accuracy and repeatability and compare it to other studies. Furthermore, we talk about advantages and limitations of the presented approach.

\section{A. Achieved Positioning Accuracy and Repeatability}

The repeatability of a PCR is evaluated in [3]. For their 6DoF spatial PCR, modeled with Cosserat theory, Orekhov et al. quantifies RMS positional repeatability as $2.1 \%$ relating to the mean continuum leg's length. Orientation RMS repeatability is specified as $1.96^{\circ}$. With RMS positioning and orientation repeatabilities of $0.3 \%$ and $0.2^{\circ}$, respectively, our prototype achieves similar results. We note that the system investigated in [3] is a more complex 6-DoF spatial design, which may lead to slightly higher errors. [3] also states positioning accuracy results. The experimental setup achieves an accuracy of $2.8 \%$ relating to mean continuum leg's length. Wu et al. determined positioning accuracy of $0.43 \%$ with their kinetostatically modeled PCR in [8] and $0.8 \%$ in [9], respectively. For the Delta continuum robot in [13], modeled based on the constant curvature approach, an accuracy of $0.8 \%-2.5 \%$ was determined (calculated as a relative value from data in the paper). Chen et al. point out a positioning accuracy of $0.45 \%$ in [30], using a discretization-based model approach. Please note that the accuracies are not directly comparable, because of different robot kinematics, control schemes, and applied models. Our resulting accuracies of $1.4 \%$ for positioning and $1.1^{\circ}$ for orientation are on par to other constant curvature approaches.

\section{B. Proposed Prototype}

The proposed prototype possesses a passive structural compliance, which allows safe human-robot-interaction or the assembly of fragile objects. In order to evaluate a constant curvature approach for PCR, the chosen design is limited to a planar case. A solely geometric model assumes a force free system, though PCR induce coupling forces into the continuum components. To determine its accuracy in a parallel setup, model evaluation excluded disturbances by additional loads or control gains, while the remaining error sources were identified. In a virtual work extension to the applied model [31], additional loads could be considered. Although, the bellow shape of the continuum segments differs significantly from common cylindrical continuum designs since the cross-sectional area varies along the backbone, resulting in bending and shear tension instead of pressure tension when loaded axially and in a complex model adaption. The current continuum design was inspired by [25], but it was up-scaled to expand its movement range and a flexible backbone was added to increase the overall stiffness. Without a support, material fatigue occurs after a few hours when solely clamped at one continuum end and orientated horizontally. Therefore, 
the PCR is supported by a base plane that introduces friction into the system. Further investigations are needed to improve an up-scaled version of the continuum robot design, enabling it to withstand loads resulting from its own gravity forces and additional external loads.

\section{CONCLUSion}

In this paper, a novel planar, active-bending parallel continuum robot is introduced. The kinematic model, based on a geometrical constant curvature approach, is calibrated by an optimization procedure to match the proposed real robotic prototype. The model is further used to optimize the size and shape of the resulting workspace. The proposed kinematic model is experimentally evaluated on the real prototype in terms of repeatability, pose accuracy and path accuracy. The achieved results lie within similar ranges as comparable PCR models in literature, which are mostly modeled using forceand torque-dependent approaches based on either beam theories or discretized lumped-element models. Our prototype achieves a mean positioning accuracy of $1.4 \%$ relating to nominal length of continuum segments. Our work demonstrates that constant curvature approach is capable to enable sufficient accuracy in absence of external forces.

In future work, we will extend our proposed kinematic model by a virtual work approach to account for external loads (compare [31]), while addressing the problem of varying model parameters along the backbone. In addition, we plan to redesign our actuation system and the continuum design to remove gear backlash and static friction on the base plane as identified error source in our system. Overall, our proposed design is the first continuum robot counterpart of a 3-RRR parallel robot. We believe that parallel continuum robots represent a valuable alternative in those application areas requiring low manipulator weights.

\section{REFERENCES}

[1] J. A. Rivera and C. J. Kim, "Spatial parallel soft robotic architectures," in IEEE/RSJ International Conference on Intelligent Robots and Systems, 2014, pp. 548-553.

[2] C. E. Bryson and D. C. Rucker, "Toward parallel continuum manipulators," in IEEE International Conference on Robotics and Automation, 2014, pp. 778-785

[3] A. L. Orekhov, C. B. Black, J. Till, S. Chung, and D. C. Rucker, "Analysis and validation of a teleoperated surgical parallel continuum manipulator," IEEE Robotics and Automation Letters, vol. 1, no. 2, pp. 828-835, 2016.

[4] A. L. Orekhov, V. A. Aloi, and D. C. Rucker, "Modeling parallel continuum robots with general intermediate constraints," in IEEE International Conference on Robotics and Automation, 2017, pp. 6142-6149.

[5] E. M. Young and K. J. Kuchenbecker, "Design of a parallel continuum manipulator for 6-DOF fingertip haptic display," in IEEE World Haptics Conference, 2017, pp. 599-604.

[6] J. Till, C. E. Bryson, S. Chung, A. Orekhov, and D. C. Rucker, "Efficient computation of multiple coupled cosserat rod models for real-time simulation and control of parallel continuum manipulators," in IEEE International Conference on Robotics and Automation, 2015, pp. 5067 5074.

[7] C. B. Black, J. Till, and D. C. Rucker, "Parallel continuum robots: Mod eling, analysis, and actuation-based force sensing," IEEE Transactions on Robotics, vol. 34, no. 1, pp. 29-47, 2018.

[8] G. Wu, G. Shi, and Y. Shi, "Modeling and analysis of a parallel continuum robot using artificial neural network," in IEEE International Conference on Mechatronics, 2017, pp. 153-158.
[9] G. Wu and G. Shi, "Experimental statics calibration of a multi-constraint parallel continuum robot," Mechanism and Machine Theory, vol. 136, pp. 72-85, 2019.

[10] C.-H. Kuo, Y.-C. Chen, and T.-Y. Pan, "Continuum Kinematics of a Planar Dual-Backbone Robot Based on Pseudo-Rigid-Body Model: Formulation, Accuracy, and Efficiency," in ASME International Design Engineering Technical Conferences and Computers and Information in Engineering Conference, 2017, pp. DETC2017-67 853.

[11] E. Shahabi and C.-H. Kuo, "Solving inverse kinematics of a planar dual-backbone continuum robot using neural network," in European Conference on Mechanism Science 2018, Cham, 2019, pp. 355-361.

[12] I. Singh, M. Singh, P. Pathak, and R. Merzouki, "Optimal work space of parallel continuum manipulator consisting of compact bionic handling arms," in IEEE International Conference on Robotics and Biomimetics, 2017, pp. 258-263.

[13] Z Yang, X. Zhu, and $\mathrm{K} . \mathrm{Xu}$, "Continuum delta robot: a novel translational parallel robot with continuum joints," in IEEE International Conference on Advanced Intelligent Mechatronics, 2018, pp. 748-755.

[14] O. Altuzarra, M. Diez, J. Corral, and F. Campa, "Kinematic analysis of a flexible tensegrity robot," New Advances in Mechanisms, Mechanical Transmissions and Robotics, vol. 46, pp. 457-464, 2017.

[15] O. Altuzarra, D. Caballero, Q. Zhang, and F. J. Campa, "Kinematic characteristics of parallel continuum mechanisms," in Advances in Robot Kinematics 2018, 2019, pp. 293-301.

[16] O. Altuzarra and J. P. Merlet, "Certified kinematics solution of 2-do planar parallel continuum mechanisms," in Advances in Mechanism and Machine Science, 2019

[17] O. Altuzarra, D. Caballero, F. J. Campa, and C. Pinto, "Forward and inverse kinematics in 2-dof planar parallel continuum manipulators," in European Conference on Mechanism Science, 2019, pp. 231-238.

[18] F. J. Campa, M. Diez, D. Diaz-Caneja, and O. Altuzarra, "A 2 dof continuum parallel robot for pick \& place collaborative tasks," in Advances in Mechanism and Machine Science, 2019, pp. 1979-1988.

[19] G. Chen, Z. Zhang, Z. Chen, and H. Wang, "A general discretizationbased approach for the kinetostatic analysis of closed-loop rigid/flexible hybrid mechanisms," in Advances in Robot Kinematics 2018, 2019, pp 269-276.

[20] C. Du, G. Chen, Z. Zhang, L. Tang, and H. Wang, "Design and experimental analysis of a planar compliant parallel manipulator," in International Conference on Intelligent Robotics and Applications. Springer, 2019, pp. 637-647.

[21] J. Burgner-Kahrs, D. C. Rucker, and H. Choset, "Continuum robots for medical applications: A survey," IEEE Transactions on Robotics, vol. 31 no. 6, pp. 1261-1280, 2015.

[22] A. A. A. Moghadam, A. Kouzani, K. Torabi, A. Kaynak, and M. Shahinpoor, "Development of a novel soft parallel robot equipped with polymeric artificial muscles," Smart Materials and Structures, vol. 24, no. 3, p. 035017,2015

[23] R. J. Webster III and B. A. Jones, "Design and kinematic modeling of constant curvature continuum robots: A review," International Journal of Robotics Research, vol. 29, no. 13, pp. 1661-1683, 2010.

[24] J.-P. Merlet, Parallel Robots, 2nd ed. Springer Netherlands, 2006.

[25] D. Kundrat, A. Schoob, T. Piskon, R. Grässlin, P. J. Schuler, T. K. Hoffmann, L. A. Kahrs, and T. Ortmaier, "Toward assistive technologies for focus adjustment in teleoperated robotic non-contact laser surgery," IEEE Transactions on medical robotics and bionics, vol. 1, no. 3, pp. 145-156, 2019.

[26] H. Edelbrunner, D. G. Kirkpatrick, and R. Seidel, "On the shape of a set of points in the plane," IEEE Transactions on Information Theory, vol. IT-29, no. 4, 1983

[27] Z. S. Roth, B. W. Mooring, and B. Ravani, "An overview of robot calibration," IEEE Robotics and Automation, vol. 3, no. 5, pp. 377-385, 1987.

[28] A. Joubair, M. Slamani, and I. A. Bonev, "Kinematic calibration of a 3dof planar parallel robot," Industrial robots, vol. 39, no. 4, pp. 392-400, 2012

[29] J. O. Rawlings, S. G. Pantula, and D. A. Dickey, Applied Regression Analysis: A Research Tool, 2nd ed. Springer New York, 1998.

[30] G. Chen, Z. Zhang, L. Kong, and H. Wang, "Analysis and validation of a flexible planar two degrees-of-freedom parallel manipulator with structural passive compliance," Journal of Mechanisms and Robotics, vol. 12 , no. 1 , p. 011011,2020

[31] N. Simaan, K. Xu, A. Kapoor, W. Wei, P. Kazanzides, P. Flint, and R. Taylor, "Design and integration of a telerobotic system for minimally invasive surgery of the throat," The International Journal of Robotics Research, vol. 28, no. 9, pp. 1134-1153, 2009. 